\title{
Relevant research article summaries
}

\author{
Nelson Fok \\ Adjunct Professor, Concordia University of Edmonton, Edmonton, AB, Canada
}

\begin{abstract}
1. Danopoulos, E., et al. 2020. Microplastic contamination of seafood intended for human consumption: A systematic review and meta-analysis. Environ. Health Perspect. 128(12): 126002.
\end{abstract}

Fifty studies were included in this systematic review of microplastic (MP). Mollusks collected off the coasts of Asia were the most heavily contaminated, coinciding with reported trends of MP contamination in the sea. MP content was $0-10.5 \mathrm{MPs} / \mathrm{g}$ in mollusks, 0.1-8.6 MPs/g in crustaceans, 0-2.9 MPs/g in fish, and $1 \mathrm{MP} / \mathrm{g}$ in echinodermata. Maximum annual human $\mathrm{MP}$ uptake was estimated to be close to 55,000 MP particles.

2. Tobias, C., et al. 2020. Plastic in global rivers: Are floods making it worse? Environ Res Lett. 16(2): 025003. doi. org/10.1088/1748-9326/abd5df

The results suggested a high inter-annual variability in plastic mobilisation, previously ignored by global plastic transport models. Flood defences reduce plastic mobilisation substantially, but regions vulnerable to flooding often coincide with high plastic mobilisation potential during floods. Consequentially, clean-up and mitigation measures and flood risk management are inherently interdependent and need to be managed holistically.

3. Collier, S. A., et al. 2021. Estimate of burden and direct healthcare cost of infectious waterborne disease in the United States. Emerg. Infect. Dis. 27(1): 140-149.

Researchers estimated the total illnesses, emergency department (ED) visits, hospitalizations, deaths, and direct health care costs for 17 waterborne infectious diseases. About 7.15 million waterborne illnesses occur annually, resulting in 601,000 ED visits, 118,000 hospitalizations, and 6,630 deaths and incurring US $\$ 3.33$ billion in direct health care costs. Otitis externa and norovirus infection were the most common illnesses. Most hospitalizations and deaths were caused by biofilm-associated pathogens (nontuberculous mycobacteria, Pseudomonas, Legionella), costing US \$2.39 billion annually.

4. Baker-Goering, M., et al. 2021. Economic burden of legionnaires' disease, United States, 2014. Emerg. Infect. Dis. 27(1): 255-257.

Researchers estimated the lifetime economic burden of 2014 Legionnaires' disease cases in the United States at $\approx \$ 835$ million. This total includes $\$ 21$ million in productivity losses caused by absenteeism and $\$ 412$ million in productivity losses caused by premature deaths.

5. Roman, L., et al. 2020. Plastic pollution is killing marine megafauna, but how do we prioritize policies to reduce mortality? Conservat. Lett., doi.org/10.1111/conl.12781

Researchers evaluated which items were responsible for the highest mortality, and which, if reduced by policy responses or other means, could result in the largest reduction in debris mortality. A limited number of consumer items were shown to be responsible for most megafauna deaths. Flexible plastic is responsible for the largest proportion of debris deaths, primarily due to gastric obstructions. Disproportionately lethal items included plastic bags/sheets/packaging, rope/fishing nets, fishing tackle, and balloons/latex. Smaller items, including "microplastics," though abundant, were seldom implicated in mortality.

6. Landrigan, P. J., et al. 2020. Human health and ocean pollution. Ann. Global Health. 86(1): 151.

Manufactured chemicals such as phthalates, bisphenol A, flame retardants, and perfluorinated chemicals, are released into the seas from plastic waste. These chemicals can disrupt endocrine signaling, reduce male fertility, damage the nervous system, and increase the risk of cancer. Harmful algal blooms (HABs) produce potent toxins that accumulate in fish and shellfish. When ingested, these toxins can cause severe neurological impairment and rapid death. HAB toxins can also become airborne and cause respiratory disease. Pathogenic marine bacteria cause gastrointestinal diseases and deep wound infections. With climate change and increasing pollution, risk is high that Vibrio infections, including cholera, will increase in frequency and extend to new areas. All the health impacts of ocean pollution fall disproportionately on vulnerable populations in the Global South, resulting in environmental injustice on a planetary scale.

7. Thomas, M., Feng, Y. 2020. Risk of foodborne illness from pet food: Assessing pet owners' knowledge, behavior, and risk perception.J. Food Protect. 83(11): 1998-2007.

Pet food has been identified as a source of pathogenic bacteria, including Salmonella and E. coli. A recent outbreak linked to Salmonella-contaminated pet treats infected $>150$ people in the United States. The mechanism by which contaminated pet food leads to human illness has not been explicated. This study was conducted to evaluate pet owners' food safety knowledge and 
pet food handling practices through an online consumer survey. Almost all pet owners (93\%) interacted with their pets, and most cuddled, allowed their pets to lick them, and slept with their pets. Less than one-third of pet owners washed their hands with soap after interacting with their pets. Over half $(58 \%)$ of the owners reported washing their hands after feeding their pets. Most pet owners fed their pets dry pet food and dry pet treats. Some fed their pets raw meat or raw animal product diets because they believed these diets to be beneficial to the pet's overall health. Many owners (78\%) were unaware of pet food recalls or outbreaks associated with foodborne pathogens. Less than $25 \%$ considered dry pet foods and treats as a potential source of foodborne pathogens. Pet owners were more concerned when seeing news about pets becoming ill from pet food and less concerned when seeing news about people becoming ill from pet food.

8. Hoover, E. R., et al. 2020. Restaurant policies and practices related to norovirus outbreak size and duration. J. Food Protect. 83(9): 1607-1618.

Data for 124 norovirus outbreaks and outbreak restaurants were obtained from Centers for Disease Control and Prevention surveillance systems and analyzed to identify relationships between restaurant characteristics and outbreak size and duration. Compared with their counterparts, restaurants that had smaller outbreaks had the following characteristics: managers received food safety certification, managers and workers received food safety training, food workers wore gloves, and restaurants had cleaning policies. In addition, restaurants that provided food safety training to managers, served food items requiring less complex food preparation, and had fewer managers had shorter outbreaks compared with their counterparts. These findings suggest that restaurant characteristics play a role in norovirus outbreak prevention and intervention.

9. Laccarino, L., et al. 2020. Association between ambient air pollution and amyloid positron emission tomography positivity in older adults with cognitive impairment. JAMA Neurol., doi:10.1001/jamaneurol.2020.3962

In this cross-sectional study of 18,178 individuals with cognitive impairment, people living in areas with worse air quality were more likely to have positive amyloid positron emission tomography scan results. Specifically, higher $\mathrm{PM}_{2.5}$ concentrations appeared to be associated with brain amyloid- $\beta$ plaques, a signature characteristic of Alzheimer's disease. This association was dose dependent and statistically significant after adjusting for demographics, lifestyles, and socioeconomic factors as well as medical comorbidities. Findings of this study suggest that exposure to air pollution is associated with risk of Alzheimer's disease and dementia.

10. Holm, S. M., et al. 2021. Health effects of wildfire smoke in children and public health tools: A narrative review. J. Expo. Sci. Environ. Epidemiol. 31: 1-20.

There are an estimated 7.4 million children in the United States affected by wildfire smoke annually, many of them in the Southeast, Pacific Northwest, and California. There is an established literature of health effects in children from components of ambient air pollution, which are also present in wildfire smoke, and an emerging literature on the effects of wildfire smoke, particularly for respiratory outcomes. Surgical masks and respirators can provide limited protection for children during wildfire events, with expected decreases of roughly $20 \%$ and $80 \%$ for surgical masks and N95 respirators, respectively. Schools should improve filtration to reduce exposure of children to smoke during wildfire events.

11. Ragusa, A., et al. 2021. Plasticenta: First evidence of microplastics in human placenta. Environ. Int. 146: 106274.

Microplastics are particles smaller than $5 \mathrm{~mm}$ deriving from the degradation of plastic objects present in the environment. In this study, six human placentas, collected from consenting women with physiological pregnancies, were analyzed to evaluate the presence of microplastics. In total, 12 microplastic fragments (ranging from 5 to $10 \mu \mathrm{m}$ in size), with spheric or irregular shape were found in 4 placentas ( 5 in the fetal side, 4 in the maternal side, and 3 in the chorioamniotic membranes). All microplastics particles were characterized in terms of morphology and chemical composition. All of them were pigmented; three were identified as stained polypropylene, a thermoplastic polymer, while for the other nine it was possible to identify only the pigments, which were all used for man-made coatings, paints, adhesives, plasters, finger paints, polymers, and cosmetics and personal care products.

12. Isaacson, K. P., et al. 2020. Drinking water contamination from the thermal degradation of plastics: Implications for wildfire and structure fire response. Environ. Sci. Water Res. Technol., doi.org/10.1039/D0EW00836B

This study was conducted to determine if the thermal degradation of various plastic drinking water pipes (i.e., PEX, HDPE, PP, PVC, and CPVC) may be a source of drinking water contamination. Widespread volatile organic compound (VOC) contamination was found in water distribution systems following three wildfires in California. A potential source of this contamination was thought to be due to the degradation of plastic components in drinking water distribution systems. Eleven plastic drinking water pipes, across eight brands, were exposed to elevated temperatures $\left(200-400^{\circ} \mathrm{C}\right)$, and subsequently submerged in water or in $n$-hexane to observe the extent of VOC leaching. Results indicated that thermally damaged drinking water pipes can be sources of VOC leaching, with 10 of the 11 materials leaching benzene, a carcinogen, into water. As exposure temperature increased, an increase in VOC leaching was observed in the polyethylene materials. Conversely, in the vinyl materials the significant mass loss associated with high exposure temperature was inversely proportional to the amount of BTEX leaching that was observed. Additional tentatively identified compounds (TICs), consisting primarily of aliphatic hydrocarbons, saturated ketones, or aromatic compounds, were found in the water (22 TICs) and n-hexane (134 TICs) leachate of burned plastics. This study has significant implications for both wildfire and structure fire recovery as plastic materials are increasingly being used in buried and 
building plumbing, and visual inspection is not a sufficient indicator of contamination risk.

13. Eldeirawi, K., et al. 2020. Increased disinfectant use among adults with asthma in the era of COVID-19.J. Allergy Clin. Immunol., doi.org/10.1016/j.jaip.2020.12.038

A cross-sectional, online survey was launched in May 2020 and data were collected from 795 US participants. More than 95\% of participants reported increased handwashing practices, whereas $89 \%$ reported increased use of alcohol-based sanitizer since the COVID-19 pandemic. The percent of participants who reported household disinfectant use $\geq 5$ times per week increased $138 \%$ for disinfectant wipes, $121 \%$ for disinfectant sprays, $155 \%$ for bleach and water solutions, and $89 \%$ for other disinfecting liquids since the COVID-19 pandemic. Household use of disinfectants $\geq 5$ times per week since the COVID-19 pandemic was higher among less educated participants, participants from small towns/rural areas, non-white, and those residing with family and/or friends. In unadjusted analyses, household use of disinfectant wipes, disinfectant sprays, bleach and water solutions, and other disinfecting liquids $\geq 5$ times per week (vs $<5$ times per week) significantly increased the odds of uncontrolled asthma. In adjusted analyses, the elevated odds ratio persisted for household use of disinfectant wipes, disinfectant sprays, bleach and water solutions, and other disinfecting liquids, although only significantly elevated for wipes and other liquids. Health care providers should recognize the potential impact of cleaning/disinfecting practices on people with asthma, particularly the use of known asthmagenics such as bleach and other disinfectants. Individuals with asthma should be provided with safer cleaning/disinfecting options such as the guidance found on the US EPA's website.

14. Bujazek, T., et al. 2021. Characterization of microplastics and anthropogenic fibers in surface waters of the North Saskatchewan River, Alberta, Canada. Facets. 6(1): 26-43.

This study investigated the occurrence, composition, and spatial trends of microplastic contamination in the North Saskatchewan River flowing through Edmonton, Alberta, the fifth largest city in Canada. Surface water samples were collected from seven sites throughout the city, upstream and downstream of the city, and near potential point sources (i.e., a wastewater treatment plant). Microplastics were found in all samples, ranging in concentration from 4.6 to 88.3 particles $/ \mathrm{m}^{3}$. Fibers were the dominant morphology recovered, and most were of anthropogenic origin and chemically identified as dyed cotton or polyester. The majority of fragments were identified as polyethylene or polypropylene. No upstream to downstream differences were found in concentration, size distribution, or morphological composition suggesting nonpoint sources of microplastics to the river. This study represents one of the first investigations into the occurrence of microplastics in the freshwater environment in western Canada. 